\title{
Prediction Intervals for Electric Load Forecast: Evaluation for Different Profiles
}

\author{
Vânia Almeida \\ LIAAD/INESC TEC \\ University of Porto \\ Porto, Portugal \\ Email: vaniagalmeida@gmail.com
}

\author{
João Gama \\ LIAAD/INESC TEC and Faculty of Economics \\ University of Porto \\ Porto, Portugal \\ Email: jgama@fep.up.pt
}

\begin{abstract}
Electricity industries throughout the world have been using load profiles for many years. The load data contains a lot of valuable knowledge, which is useful for both electricity producers and consumers. Currently, PIs are assuming increasing importance comparatively to point forecast. The key point is the compromise between informativeness and correctness. This paper aims to evaluate neural network-based models to interval forecast of load demand. We aim to demonstrate that demand profiles clearly influence prediction intervals (PIs) reliability and width. A real data of aggregated load demand constituted by different customer profile classes is used. The evaluation is performed for different load profiles that are identified on the basis of their electricity behavior using hierarchical clustering, and taking the Kullback-Leibler divergence as the distance metric. PIs are compared using two different strategies: (1) dual perturb and combine (DPC) algorithm and (2) conformal prediction. Comparative results show that PI models performance is significantly different for different load profiles. The knowledge retrieved from load pattern analysis can support the selection of the method of producing interval forecasts for a specific location. An also, support the selection of an optimum confidence level, considering that a too wide PI conveys little information and is of no use for decision making.
\end{abstract}

Keywords: load forecasting, prediction intervals, hierarchical clustering, Kullback-Leibler divergence

\section{INTRODUCTION}

Electricity industries throughout the world have been using load profiles for many years. A load profile describes the pattern of electricity usage for a customer or a group of customer over a given period. This information is valuable for electricity suppliers that aim to get better knowledge of their customers and customize their supply strategies. But, it is also valuable knowledge for consumers that became aware of their consumer behavior [1].

There is a great difference in the electricity consumption patterns of different types of users, such as domestic, commercial, industrial, agricultural, etc. Most current research work focuses on clustering load data of industrial customers other than ordinary residential customers [2]. However, domestic load occupies a large part of total electricity consumption. So, clustering analysis of residential customers is of great significance. Domestic load is usually not as stable as industrial consumption. Contrasting with industrial load clustering analysis that uses the Euclidean distance as the distance measurement, residential load data requires a different metric.
Load forecasting is a fundamental and important task to operate the power system efficiently and economically. It can be classified into different categories according to the forecast horizon (short-term, medium-term, and long-term), and in terms of forecasting outputs, categorized into point forecasts or prediction intervals (PIs). Point forecasts cannot properly handle uncertainties associated with datasets [3]. PIs are an interesting option, since more than provide accurate forecasts, reliable interval predictions are fundamental [4]. By definition, a PI is an estimate of an interval in which a future observation will fall, with a certain probability called confidence level. Typically, PIs evaluation is focused on the calibration of confidence intervals that indicates the probability for correct predictions. But, the confidence value cannot be considered individually. Higher probability values are associated to intervals that can include extreme prediction errors. And so, a too wide PI conveys little information and is of no use for decision making.

The knowledge retrieved from load pattern analysis can be used to improve the accuracy of load forecasting. This information can be used to support the accurate forecasting of load in many ways. Wijaya et al. [5] demonstrated that forecasting each cluster separately and then aggregating the forecast performs better than forecast directly the aggregate load). Hernandez et al. [6] presented a solution for shortterm load forecasting in microgrids, based on a three-stage architecture which starts with pattern recognition by a selforganizing map, clustering of the previous partition via kmeans algorithm, and at end performs demand forecasting for each cluster with a multilayer perceptron. Jota et al. [7] gave a load forecasting method of daily load curves and the peak load based on the typical daily curve obtained by hierarchical clustering. Using information from residential demand profiles, Rhodes et al. (2014) studied the optimal number of representative residential electricity use profiles within each season, and draw correlations to the different profiles based on data from 103 homes [8].

This paper aims to evaluate neural network (NN)-based models to interval forecast of load demand for different load profiles. The load profiles are identified on the basis of their electricity behavior using hierarchical clustering, and taking the Kullback-Leibler divergence as the distance metric. PIs are compared using two different strategies, (1) dual perturb and combine (DPC) algorithm and (2) conformal prediction. The paper is organized as follows. Sections II and III describe 
related work on clustering, and electric load forecasting, respectively. The proposed methodology is formulated in Section IV. Case study description and results are presented in Section V. Finally, Section VI concludes this paper and provides guidelines for future work.

\section{Clustering LOAD DATA}

Clustering methods [9] can be grouped into different categories based on the clustering criterion. Zhou et al. [1] provide an overview of some clustering methods which have been used for creating electricity load profile classes. A brief comparison traditional clustering algorithms, such as K-means, FCM (Fuzzy c-means) and hierarchical method is made. In this study we used hierarchical clustering that allows us to determine the numbers of clusters flexibly based on granularity of the obtained clusters.

\section{A. Dissimilarity Measures}

As most of the clustering techniques requires a measure of distance between the objects to be clustered. The choice of the distance has a preponderant influence on the result of cluster analysis. Typically, Euclidean distance is the preferred metric [10]. Other distances can be used, such as Dynamic Time Warping (DTW) that groups time series based on their shape information. It is indicated to cluster time series exhibiting similar patterns at different time periods [11]. Hino et al. [12] demonstrated that using the symmetrizing generalized Kullback-Leibler divergence as a distance measure of the distributions, typical patterns of the consumption can be extracted by hierarchical clustering.

In this work, load historical data are represented as histograms. So, the desired distance should be based on correspondences between bins in the histograms. A drawback of bin-by-bin dissimilarity measures is their sensitivity to bin size. A binning that is too coarse will not have sufficient discriminative power, while a binning that is too fine will place similar features in different bins which will never be matched. Several measures can be employed [13]. In this study Kullback-Leibler divergence is used as distance metric. It is defined as:

$$
d_{K L}(H, K)=\sum h i \log \frac{h i}{k i}
$$

\section{ELECTRIC LOAD FORECASTING}

There are many interesting methodologies that can be used to load forecast. The use of time series analysis techniques is well accepted. Typically, load time series have three seasonal cycles: a daily cycle, a weekly cycle, and a yearly seasonal cycle. The load curve of weekend days, normally different from that of working days, can affect load curves of Mondays and Fridays, therefore the former ones are often separately treated.

Amongst the most popular methods for load forecasting are NN and ARIMA models [14]. Hippert et al. [15] present an interesting review of NNs for short-term load forecasting. Nowadays, PIs are assuming increasing importance comparatively to point forecasts, since more than provide accurate forecasts, reliable interval predictions are need.

\section{A. Prediction Intervals}

Several strategies can be used to provide PIs. Two strategies are adopted in this work: (1) dual perturb and combine (DPC) algorithm [16] which produces PIs based on the perturbed predictions, and (2) conformal prediction, one of the most promising strategies used to determine precise levels of confidence. Other strategies can be used, such as detailed in [17], [18].

1) Dual Perturb and Combine Method: DPC is an efficient method that allows the reduction of the variance exhibited by NNs, but also the estimation of confidence values of the predictions [16]. It consists of perturbing each test example several times, adding white noise to the attribute values, and predicting each perturbed version of the test examples. The final prediction is obtained by aggregating all the predictions. It is implemented as follows:

For each input variable in the test set $x, k$ perturbations are performed, $i=1, \ldots, k$.

$$
x_{i}=x+\delta_{i}
$$

with $\delta_{i}$ white noise $\mathrm{N}\left(0, \sigma_{i}^{2}\right)$, where $\sigma_{i}$ and $k$ are userdefined parameters.

$k$ predictions $\hat{y}_{i}$ are obtained, and the final prediction $\hat{y}$ is:

$$
\hat{y}=\frac{\sum \hat{y}_{i}}{k}
$$

The lower and upper bounds are defined as:

$$
\left[\min \left(\hat{y}_{i}\right), \max \left(\hat{y}_{i}\right)\right]
$$

2) Conformal Prediction: CP uses the past experience to determine precise levels of confidence in new predictions, assuming that the data is identically and independently distributed (i.i.d). CPs have been developed based on several algorithms, such as Support Vector Machines [19], k-Nearest Neighbors [20] or Neural Networks Regression [21]. A neural networks regression based on inductive conformal prediction (NNR-ICP) is implemented as proposed by Papadopoulos and Haralambous [21]:

The training and the calibration set are represented as:

- Training: $\left\{\left(x_{1}, y_{1}\right),\left(x_{2}, y_{2}\right), \ldots,\left(x_{m}, y_{m}\right)\right\} \quad$ where $m<l$

- Calibration: $\left\{\left(x_{m+1}, y_{m+1}\right),\left(x_{m+2}, y_{m+2}\right), \ldots,\left(x_{l}, y_{l}\right)\right\}$ with $k=l-m$ elements

A nonconformity score is associated with every pair $\left(x_{m+i}, y_{m+i}\right)$ in the calibration set. It evaluates how strange the pair is for the trained NNR rule, being defined as:

$$
\alpha_{i}=\left|\hat{y}_{m+1}-y_{m+1}\right|
$$

where $\hat{y}_{m+1}$ is the predicted value. 
Assuming i.i.d. distribution, these $\alpha$ 's are sort in descending order:

$$
\alpha_{m+1}, \ldots, \alpha_{m+k}
$$

Finally, the lower and upper bounds are computed according to:

$$
\left(\hat{y}_{l+1}-\alpha_{m+s}, \hat{y}_{l+1}+\alpha_{m+s}\right)
$$

where $s=\delta(k+1)$

Assuming that a confidence level, $1-\delta$, is given a priori, where $\delta>0$ is a small constant (e.g. $1 \%$ or $5 \%$ ). It means that for a $\alpha=0.05$ and a confidence level of $95 \%$, the interval width is given by $\alpha_{m+0.05(k+1)}$, where $\mathrm{k}$ is the calibration set length.

\section{CAse Study and Experimental Setup}

The methodology herein proposed is divided into two steps. The first one comprises the analysis of historical load curves and the detection of typical load curves to represent the energy use by means of cluster analysis. Secondly, 24h PIs load forecasts based on NNs are generated. Some of the techniques are detailed in sections above. Based on the output of two phases, PI width and reliability are analyzed.

\section{A. Dataset}

The dataset consists of aggregated load demand from 1 April to 31 November 2014, collected in the Customer Load Active System Services (CLASS) Project run by the UK Distribution Network Operator Electricity North West Limited ${ }^{1}$. Forty-five substations comprising different consumer load profiles are analyzed. Each one is treated individually, being $70 \%$ of data used for learning the global model, and the remaining $30 \%$ for prediction. All experiments for both algorithms were repeated 5 times.

\section{B. Hierarchial Clustering with Kulback-Leibler distance}

Load historical data are represented as histograms, setting bin number fixed to 65 . Kullback-Leibler divergence is computed using entropy $R$ package between bin counts of histograms. Cluster analysis is performed using hclust $R$ package (that includs Ward's method for hierarchical clustering).

\section{Inputs of the Neural Network}

The predictive model for the next day (hourly predictions) is a feed-forward NN. The week seasonality is considered, since the load curve of weekend days, normally different from that of working days. The choice of the network topology and inputs was motivated by previous work [22], [23], [6]. It is constituted by:

\section{Inputs:}

- 24 values of the load curve $[L(d-1) 1, L(d-$ $1) 2, \ldots, L(d-1) 24]$ of day $d-1$ (day before the forecasting day $\mathrm{d}$ ).

\footnotetext{
${ }^{1}$ https://www.enwclass.nortechonline.net/data\#substation-group/31
}

- Day of week, entered as two different variables, in the form of sines and cosines, by means of $\sin [(2 \pi d) / 7]$ and $\cos [(2 \pi d) / 7]$, for each one of the days: $\operatorname{Sunday}(d=0), \operatorname{Monday}(d=1)$, Tuesday $(d=$ $2)$, Wednesday $(d=3)$, Thursday $(d=4)$, Friday $(d=$ $5)$, Saturday $(d=6)$.

Output: 24 values of the load curve $[L d 1, \ldots, L d 24]$

\section{PIs metrics}

The literature offers a variety of methods for the evaluation of the performance of point prediction methods, e.g. mean square error (MSE), mean absolute error (MAE) or mean absolute percentage error (MAPE). However, there is no wellestablished error dedicated to the PI assessment. Typically, the evaluation is only made based on the PI coverage probability (PICP), that can be interpreted as the probability that target values will be covered by the interval bounds. It is defined as:

$$
P I C P=\frac{1}{N} \sum_{i=1}^{N} c(i)
$$

where $\mathbf{N}$ is the number of samples and $c(i)=1$, if $\hat{y}(i) \in[L(i), U(i)], \mathrm{L}(\mathrm{i})$ is the lower bound, and $\mathrm{U}(\mathrm{i})$ is the upper bound, otherwise $c(i)=0$. Ideally, the PICP should be as close as possible to its nominal value $(1-\alpha) \%$, the confidence level for which PIs have been constructed. However, without inclusion of their length the PI evaluation sound more subjective than objective. Therefore, it is essential the computation of width-based indices. Along this work, interval width is defined as the difference in amplitude between the upper and lower bound.

\section{EXPERIMENTAL RESULTS}

\section{A. Electrical load profiles}

Figure 1 shows power demand variation for the 45 substations represented by boxplot graphic. Mean power demand varies between 1 and 7.7MW. Additionally, boxplot summarizes median, upper and lower quartiles and minimum and

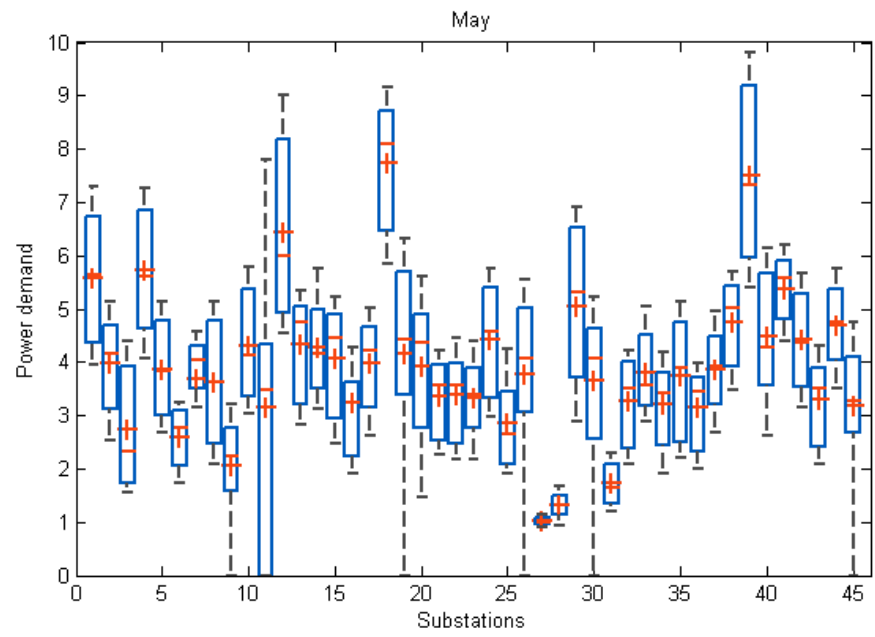

Fig. 1. Boxplot of 45 substation during May 2014. 

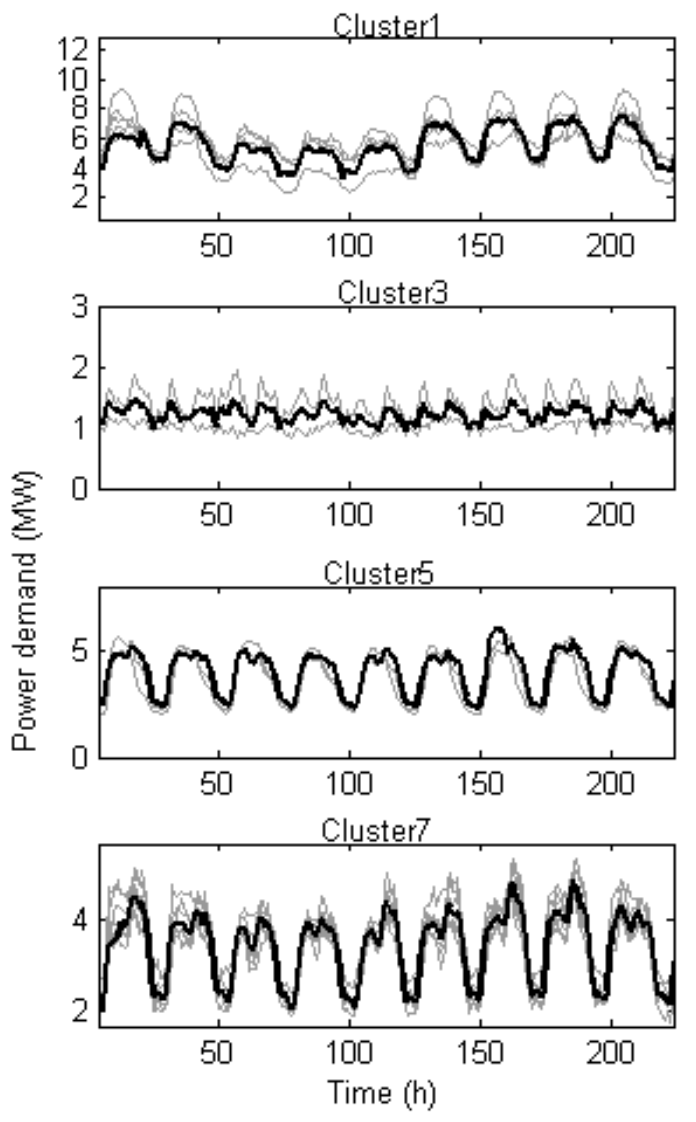

Fig. 2. Clustering of the time series for power demand.

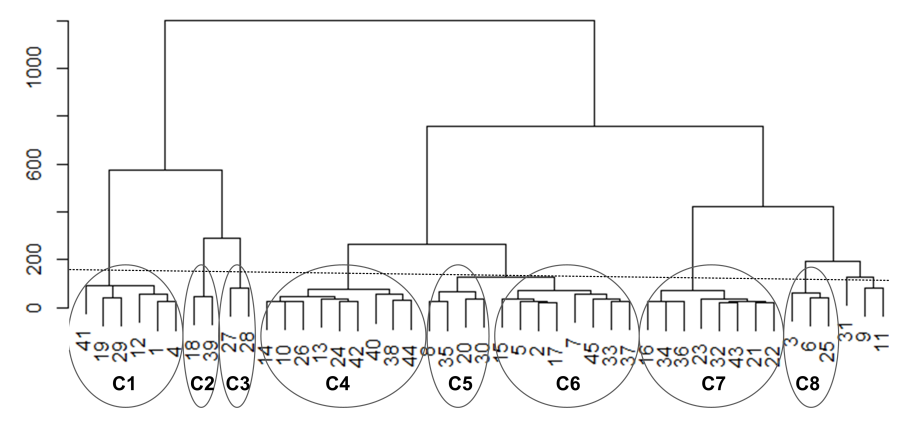

Fig. 3. Dendogram of power demand during May 2014.

maximum values. The measurement sites have different classes of customers connected to the corresponding substations, e.g. substation 29 Kitt Green (4405 customers) is largely constituted industrial and commercial substations, while substation 16 Egremont (10168 customers) is largely constituted by domestic substations [24].

The result of the cluster analysis is represented by the Dendrogram graphic, as shown in Fig. 3. The ordinate axis indicates the distance between clusters. Eight clusters are formed using data from May 2014. Future studies are required to evaluate seasonality variations along seasons. Depending of the substation type (mainly for largely domestic substations)
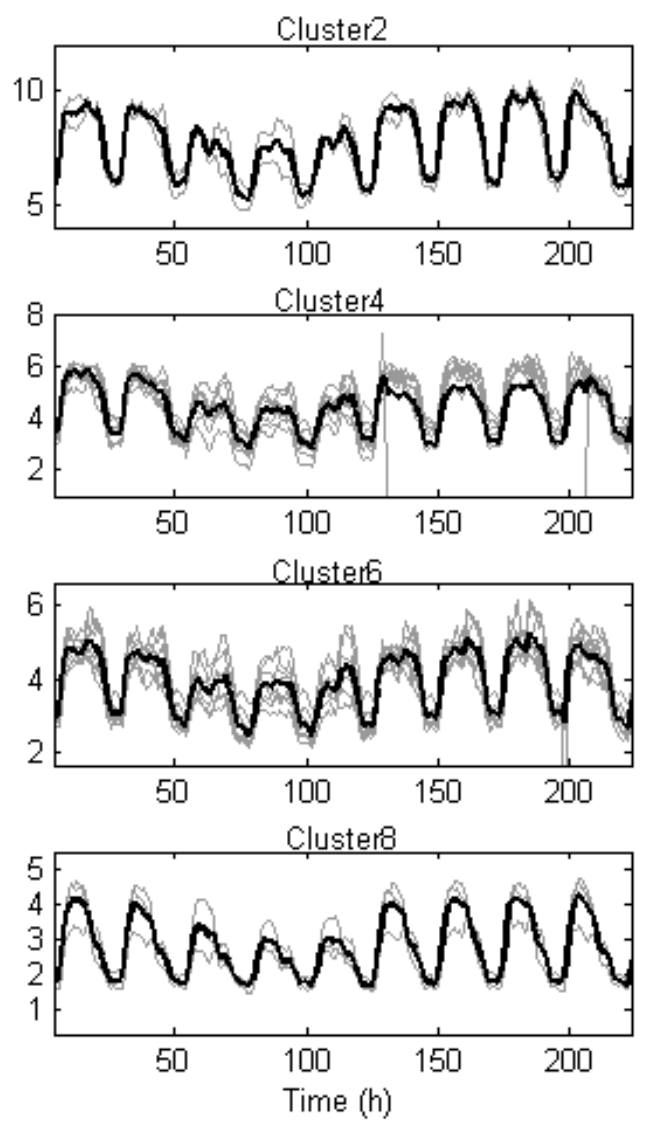

modifications are expected.

Clusters intend to group similar load profiles, as well as identify abnormally behaving. In Figure 2 different load profiles are represented, representing each one of clusters between 1st-9th May 2014. Cluster 3 represents non-typical load patterns, identified for substations 27 and 28. Cluster 2 is characterized by high power demands, in oppositions to Clusters 5-8. The first Monday of May is a bank holiday in the United Kingdom (5th May). This date also coincides with labor day (International Workers' Day). During this day is visible a decrease of power demand for clusters 1, 2, 4, 6, 8. Similarly, to the demand variation observed during weekend 3rd-4th May

\section{B. Prediction Intervals}

PIs are constructed, as described in Section III. The NNRICP model is calibrated through Equation 6. In opposition to the NNR-ICP model, the DPC method is not calibrated a priori. The jit added to the input variables follows $x_{i} \approx N\left(0, \sigma_{i}^{2}\right)$, $i=1, \ldots, 10$.

In Figure 4 are shown PIs at different confidence values for both algorithms. As expected, as the confidence level increases, the corresponding interval width is enlarged. It is also visible that NNR-ICP produces symmetric intervals, while DPC intervals are asymmetric. 

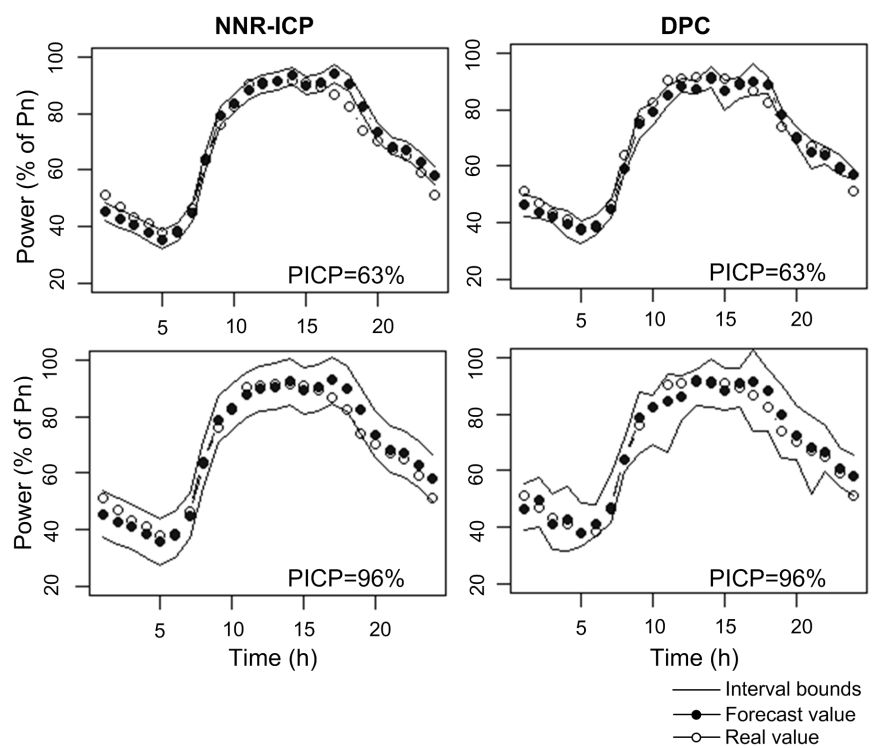

Fig. 4. PIs for the NNR-ICP and DPC methods at different condence values.

\section{PIs evaluation}

We are interested in compare the reliability and width of intervals for each one of the clusters. NNR-ICP prediction regions at different significant levels are compared in Figure 5. Cluster 5 performs differently from all other clusters. This cluster is characterized by lower reliability values compared to the expected reliability. Clusters 1 and 4 also tend to underestimate expected reliability. In opposition, clusters 2,8 tend to overestimate the reliability values. As shown, for clusters 3 and 6 and 7 the calibration fit is linear with predictions falling near to the line of equality for the predicted and expected values. And in this case, we can concluded that NNR-ICP is well calibrated in the case of the database considered in this study.

The calibration curve for DPC algorithm is shown in Figure 6. Results are presented individually for each substation,

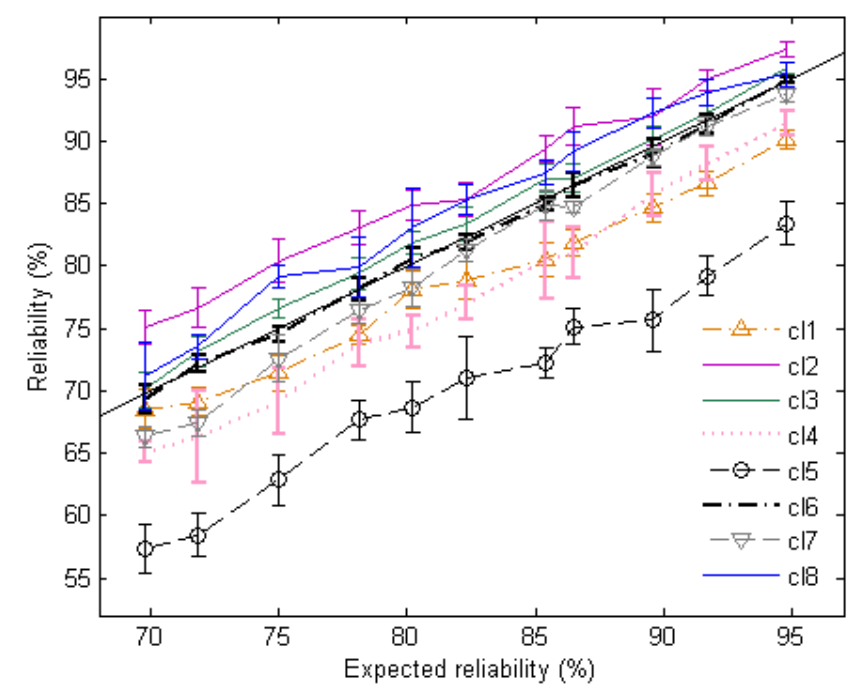

Fig. 5. NNR-ICP calibration considering each one of the clusters.

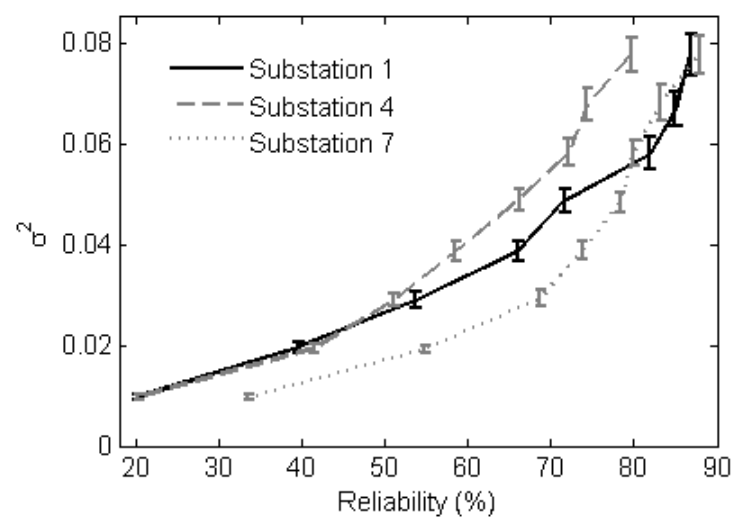

Fig. 6. DPC calibration for three different substations.
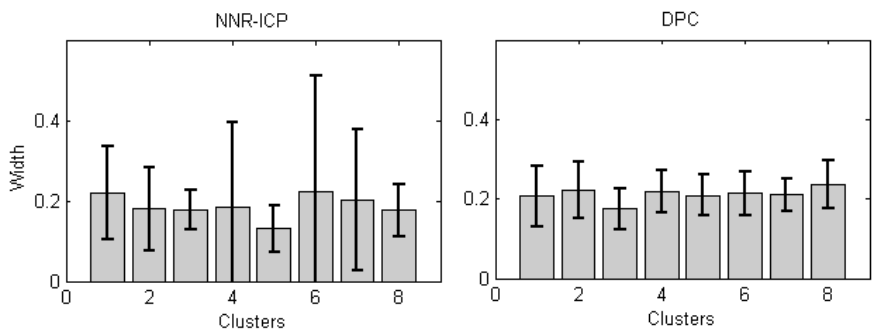

Fig. 7. PI width for each cluster obtained with NNR-ICP and DPC.

considering 5 independent trials. Calibration curves for three of the substations are depicted, evidencing the inter-substation variability. So, reliability comparisons between clusters are not performed.

In Figure 7 are represented PIs mean width (and standard deviation) for each one of the clusters during May-September, considering a reliability $\approx 82 \%$. Comparing both algorithms, PIs obtained with DPC are larger than PIs constructed with DPC. DPC interval width is similar for all clusters. In opposition NNR-ICP performs differently for each one of the clusters. Cluster 6 is characterized by intervals whose lengths are significantly larger and highly variable than PIs observed for clusters 3, 5 and 8 . The paid cost is lower informativeness. Wilcoxon test was applied, and the corresponding p-values are shown (considering a significance level of 5\%) in Tables I and II. For both cases, significant differences among groups are verified. PIs width obtained with NNR-ICP for the clusters 1,3 and 8 are significantly different from all other clusters, such as PIs obtained with DPC for the clusters 1 and 5.

From the analysis of both aspects we can conclude: 1) Cluster 5 is not well calibrated for the NNR-ICP. In this case, the use of methods capable to produce wider intervals can be benefit, such as DPC. 2) PI width for the Cluster 6 and 7 is highly variable. However, this cluster is well calibrated. The seasonal effects should be studied, since they can be in the origin of clusters reorganization during summer. 3) It is also interesting observe that in spite of Cluster 3 presets a nontypical load pattern, the reliability values observed are well calibrated (in the case of NNR-ICP). And, width values are comparable for both algorithms. 
TABLE I. WILCOXON TEST (P-VALUES) FOR PI WIDTH - NNR-ICP.

\begin{tabular}{l|l|l|l|l|l|l|l} 
& $\mathrm{C} 2$ & $\mathrm{C} 3$ & $\mathrm{C} 4$ & $\mathrm{C} 5$ & $\mathrm{C} 6$ & $\mathrm{C} 7$ & $\mathrm{C} 8$ \\
\hline C1 & $<0.05$ & $<0.05$ & $<0.05$ & $<0.05$ & $<0.05$ & $<0.05$ & $<0.05$ \\
C2 & - & $<0.05$ & 0.59 & 0.85 & 0.85 & 0.40 & $<0.05$ \\
C3 & & - & $<0.05$ & $<0.05$ & $<0.05$ & $<0.05$ & $<0.05$ \\
C4 & & & - & 0.48 & 0.15 & $<0.05$ & $<0.05$ \\
C5 & & & & - & 0.47 & $<0.05$ & $<0.05$ \\
C6 & & & & & - & 0.13 & $<0.05$ \\
C7 & & & & & & - & $<0.05$ \\
C8 & & & & & & & -
\end{tabular}

TABLE II. WILCOXON TEST (P-VALUES) FOR PI WIDTH - DPC.

\begin{tabular}{l|l|l|l|l|l|l|l} 
& $\mathrm{C} 2$ & $\mathrm{C} 3$ & $\mathrm{C} 4$ & $\mathrm{C} 5$ & $\mathrm{C} 6$ & $\mathrm{C} 7$ & $\mathrm{C} 8$ \\
\hline C1 & $<0.05$ & $<0.05$ & $<0.05$ & $<0.05$ & $<0.05$ & $<0.05$ & $<0.05$ \\
C2 & - & $<0.05$ & 0.37 & $<0.05$ & 0.37 & 0.30 & 0.09 \\
C3 & & - & $<0.05$ & $<0.05$ & 0.21 & $<0.05$ & 0.38 \\
C4 & & & - & $<0.05$ & $<0.05$ & $<0.05$ & $<0.05$ \\
C5 & & & & - & $<0.05$ & $<0.05$ & $<0.05$ \\
C6 & & & & & - & 0.18 & 0.52 \\
C7 & & & & & & - & $<0.05$ \\
C8 & & & & & & & -
\end{tabular}

\section{DISCUSSION AND CONCLUSIONS}

This paper proposes NN-based models for short-term interval load forecasting. PIs are obtained with two algorithms, DPC and NNR-ICP using NNs. The proposed methodology has been applied in real data of aggregated load demand constituted by different customer profile classes. The key outputs from this study is to demonstrate that demand profiles clearly influence PIs reliability and width using different models for interval load forecasting. The knowledge from demand profiles, can support the selection of the method of producing interval forecasts for a specific location. And also adjust an optimum confidence level, considering that a too wide PI conveys little information and is of no use for decision making.

Future studies will include seasonal profiles. Additionally, it is required a detailed analysis of weekdays and workdays profiles. In other hand, it will be interesting the study of domestic load profiles. Typical pattern of individual domestic loads is very different from the pattern of aggregated loads, in particular, the occurrence of occasional high loads, which have a magnitude equal to many multiples of the mean value.

\section{ACKNOWLEDGMENT}

This work was supported by NORTE-07-0124-FEDER000056 financed by ON.2-O Novo Norte, under the National Strategic Reference Framework, through the Development Fund, and by national funds, through FCT. And, by European Commission through MAESTRA (ICT-2013-612944).

\section{REFERENCES}

[1] K. le Zhou, S. lin Yang, and C. Shen, "A review of electric load classification in smart grid environment," Renewable and Sustainable Energy Reviews, vol. 24, no. 0, pp. 103 - 110, 2013.

[2] A. Kamilaris, B. Kalluri, S. Kondepudi, and T. K. Wai, "A literature survey on measuring energy usage for miscellaneous electric loads in offices and commercial buildings," Renewable and Sustainable Energy Reviews, vol. 34, no. 0, pp. 536 - 550, 2014.

[3] H. Quan, D. Srinivasan, and A. Khosravi, "Uncertainty handling using neural network-based prediction intervals for electrical load forecasting," Energy, vol. 73, no. 0, pp. 916 - 925, 2014.

[4] A. Khosravi, S. Nahavandi, and D. Creighton, "Construction of optimal prediction intervals for load forecasting problems," Power Systems, IEEE Transactions on, vol. 25, pp. 1496-1503, Aug 2010.
[5] T. K. Wijaya, S. F. R. J. Humeau, M. Vasirani, and K. Aberer, "Residential Electricity Load Forecasting: Evaluation of Individual and Aggregate Forecasts," tech. rep., Lausanne, Switzerland, 2014.

[6] L. Hernndez, C. Baladrn, J. M. Aguiar, B. Carro, A. Snchez-Esguevillas, and J. Lloret, "Artificial neural networks for short-term load forecasting in microgrids environment," Energy, vol. 75, no. 0, pp. 252 - 264, 2014.

[7] P. R. Jota, V. R. Silva, and F. G. Jota, "Building load management using cluster and statistical analyses," International Journal of Electrical Power Energy Systems, vol. 33, no. 8, pp. 1498 - 1505, 2011.

[8] J. D. Rhodes, W. J. Cole, C. R. Upshaw, T. F. Edgar, and M. E. Webber, "Clustering analysis of residential electricity demand profiles," Applied Energy, vol. 135, no. 0, pp. $461-471,2014$.

[9] R. Xu and I. Wunsch, D., "Survey of clustering algorithms," Neural Networks, IEEE Transactions on, vol. 16, pp. 645-678, May 2005.

[10] G. Chicco, "Overview and performance assessment of the clustering methods for electrical load pattern grouping," Energy, vol. 42, no. 1, pp. $68-80,2012$. 8th World Energy System Conference, \{WESC 2010.

[11] H. Izakian, W. Pedrycz, and I. Jamal, "Fuzzy clustering of time series data using dynamic time warping distance," Engineering Applications of Artificial Intelligence, vol. 39, no. 0, pp. 235 - 244, 2015.

[12] H. Hino, H. Shen, N. Murata, S. Wakao, and Y. Hayashi, "A versatile clustering method for electricity consumption pattern analysis in households," Smart Grid, IEEE Transactions on, vol. 4, pp. 1048-1057, June 2013.

[13] Y. Rubner, C. Tomasi, and L. Guibas, "The earth mover's distance as a metric for image retrieval," International Journal of Computer Vision, vol. 40, no. 2, pp. 99-121, 2000.

[14] S. Pappas, L. Ekonomou, P. Karampelas, D. Karamousantas, S. Katsikas, G. Chatzarakis, and P. Skafidas, "Electricity demand load forecasting of the hellenic power system using an \{ARMA\} model," Electric Power Systems Research, vol. 80, no. 3, pp. 256 - 264, 2010.

[15] H. Hippert, C. Pedreira, and R. Souza, "Neural networks for shortterm load forecasting: a review and evaluation," Power Systems, IEEE Transactions on, vol. 16, pp. 44-55, Feb 2001.

[16] P. Geurts and L. Wehenkel, "Closed-form dual perturb and combine for tree-based models," in Machine Learning, Proceedings of the TwentySecond International Conference (ICML 2005), Bonn, Germany, August 7-11, 2005, pp. 233-240, 2005.

[17] H. Quan, D. Srinivasan, and A. Khosravi, "Short-term load and wind power forecasting using neural network-based prediction intervals," Neural Networks and Learning Systems, IEEE Transactions on, vol. 25, pp. 303-315, Feb 2014.

[18] A. Khosravi, S. Nahavandi, D. C. Creighton, and A. F. Atiya, "Comprehensive review of neural network-based prediction intervals and new advances.," pp. 1341-1356, 2011.

[19] C. Saunders, A. Gammerman, and V. Vovk, "Transduction with confidence and credibility," in In Proceedings of the International Joint Conference on Artificial Intelligence, pp. 722-726, 1999.

[20] K. Proedrou, I. Nouretdinov, V. Vovk, and A. Gammerman, "Transductive confidence machines for pattern recognition," in in ECML 2002, pp. 381-390, Springer, 2001.

[21] H. Papadopoulos and H. Haralambous, "Reliable prediction intervals with regression neural networks," Neural Networks, vol. 24, no. 8, pp. $842-851,2011$. Artificial Neural Networks: Selected Papers from \{ICANN 2010.

[22] J. Gama and P. Rodrigues, "Stream-based electricity load forecast," in Knowledge Discovery in Databases: PKDD 2007, vol. 4702 of Lecture Notes in Computer Science, pp. 446-453, Springer Berlin Heidelberg, 2007.

[23] P. P. Rodrigues and J. a. Gama, "A system for analysis and prediction of electricity-load streams," Intell. Data Anal., vol. 13, pp. 477-496, Aug. 2009.

[24] A. Howard, S. Stott, P. Turner, and V. Turnham, "Interim profile modelling study," tech. rep., UK, 2015. 